\title{
ANÁLISE BIOMECÂNICA DE MEMBROS INFERIORES EM MULHERES ACOMETIDAS COM DORES ARTICULARES NO JOELHO
}

\author{
BIOMECHANICAL ANALYSIS OF INFERIOR LIMBS IN WOMEN AFFECTED \\ WITH ARTICULAR PAINS IN THE KNEE
}

\author{
Danilo Augusto Ferreira de Lima ${ }^{1}$ \\ Ronan Campos Abdon ${ }^{1}$ \\ Mariana Gouveia Gabriel ${ }^{2}$ \\ Mônica Cardoso Cruz3 \\ Biatriz Araújo Cardoso 4 \\ George Alberto da Silva Dias ${ }^{5}$ \\ Ana Júlia Cunha Brito ${ }^{6}$
}

\section{RESUMO}

Objetivo: analisar a biomecânica de membros inferiores em mulheres acometidas com dores articulares no joelho. Metodologia: Estudo observacional analítico e descritivo do tipo transversal, com 40 mulheres entre 18 a 60 anos com dor no joelho. Utilizou-se ficha de avaliação contendo as variáveis antropométricas e postural, concomitante avaliação estática e dinâmica do joelho. Analisou-se os dados pelo software BioEstat 5.0 com nível a de significância de $5 \%(p \leq 0,05)$. Resultados: Foram coletadas 40 mulheres (idade: $45,2 \pm 14,7$ anos, peso: $70,3 \pm 12,3 \mathrm{~kg}$, altura: $1,60 \pm 0,05 \mathrm{~cm}$, Índice de Massa Corpórea (IMC): $27,27 \pm 4,6$, e tamanho do pé: $36,5 \pm 1,5 \mathrm{~cm}$ ), onde o joelho direito foi o mais acometido por lesão $(70 \%)$. Observou-se que o comprimento do membro inferior direito (MID) foi $83,5 \pm 5,86$ $\mathrm{cm}$, membro inferior esquerdo (MIE) $83,4 \pm 5,89 \mathrm{~cm}$, ângulo $Q$ do MID 25,2 $\pm 6,09$ graus, ângulo $Q$ do MIE $25,3 \pm 6,57$ graus e inclinação pélvica de $1,20 \pm 0,89 \mathrm{~cm}$, onde 25 mulheres $(62,5 \%)$ apresentaram inclinação a esquerda. Para avaliação dinâmica houve positividade do teste Step Down de forma significativa apenas para o MID (77,5\%; $p=0,0009)$. Na associação das variáveis estudadas com teste de Step Down, houve significância estatística para peso (MID: $p=0,05$ e MIE: $p=0,007$ ), bem como tamanho do pé do MID ( $p=0,04)$, para o IMC apenas o MIE $(p=0,01)$. $E$, na relação dos dados posturais houve significância estatística para o comprimento do MID $(p=0,01)$. Conclusão: As variáveis peso, IMC, tamanho do pé e comprimento do membro inferior tiveram relação sobre o quadril e os MMII, sugerindo valgo dinâmico e/ou predisposição do agravamento de dores no joelho em mulheres.

DESCRITORES: Genu Valgum. Movimento. Mulheres.

\begin{abstract}
Objective: to analyze the biomechanics of lower limbs in women with joint pain in the knee. Methodology: Observational descriptive and cross-sectional study with 40 women between 18 and 60 years with knee pain. An evaluation form containing the anthropometric and postural variables, concomitant static and dynamic evaluation of the knee was used. Data was analyzed by BioEstat 5.0 software with an a level of significance of $5 \%$ $(p \leq 0.05)$. Results: A total of 40 women were gathered (age: $45.2 \pm 14.7$ years, weight: $70.3 \pm 12.3 \mathrm{~kg}$, height: $1.60 \pm 0.05$ $\mathrm{cm}$, Body Mass Index (BMI): $27.27 \pm 4.6$, and foot size: $36.5 \pm$ $1.5 \mathrm{~cm}$ ), where the right knee was the most affected by injury $(70 \%)$. It was observed that the length of the right lower limb (RLL) was $83.5 \pm 5.86 \mathrm{~cm}$, left lower limb (LLL) $83.4 \pm 5.89$ $\mathrm{cm}, \mathrm{Q}$ angle of the RLL $25.2 \pm 6.09$ degrees , LLL $\mathrm{Q}$ angle of $25.3 \pm 6.57$ degrees and pelvic tilt of $1.20 \pm 0.89 \mathrm{~cm}$, where 25 women $(62.5 \%)$ presented left slope. For dynamic evaluation, the Step Down test was positively significant only for the RLL $(77.5 \%, p=0.0009)$. In the association of the variables studied with the Step Down test, there was statistical significance for weight (RLL: $p=0.05$ and LLL: $p=0.007$ ), as well as foot size of the RLL $(p=0.04)$, for BMI only the LLL $(p=0.01)$. And, in the relation of the postural data there was statistical significance for the length of the RLL $(p=0.01)$. Conclusion: The variables weight, BMI, foot size and lower limb length had relation over the hip and LLL, suggesting dynamic valgus and / or predisposition for worsening of knee pain in women.
\end{abstract}

DESCRIPTORS: Genu Valgum. Movement. Women.

1- Acadêmico do curso de fisioterapia da Universidade da Amazônia (UNAMA), Belém - PA.

2- Fisioterapeuta. Especialista em Fisioterapia Motora Aplicada a Ortopedia e Traumatologia-UNIFESP.

3- Fisioterapeuta. Mestra em Fisioterapia (UNIMEP)

4- Fisioterapeuta. Doutora em Doença Medicina Tropical/FIOCRUZ. Professora da Universidade da Amazônia (UNAMA).

5- Fisioterapeuta. Doutor em Doenças Tropicais pela Universidade Federal do Pará (UFPA). Professor da Universidade do Estado do Pará (UEPA).

6- Fisioterapeuta. Mestre em Desenvolvimento e Meio Ambiente Urbano pela Universidade da Amazônia (UNAMA). Professora da Faculdade Metropolitana da Amazônia (FAMAZ). 
A s dores articulares podem ser consideradas um dos sintomas Traumato-ortopédicos mais frequentes no joelho e suas etiologias demonstram estar ligadas a causas multifatoriais, dificultando a caracterização dos indivíduos com a disfunção'.

Como se sabe, esse problema tem como principal particularidade a presença de algia na região do joelho, porém, quando é associada a atividades que provocam o aumento da compressão e do cisalhamento nas articulações, como agachar ou subir e descer degraus, a sintomatologia tende a ficar mais perceptível devido ao aumento da dor e a crepitação no segmento².

Esta condição abrange em maior porcentagem mulheres do que homens, sendo as praticantes de atividade física com maior tendência para esse problema, uma vez que submetem o complexo do joelho a sobrecargas biomecânicas predispondo ao aumento do ângulo $Q$ e da frouxidão articular fisiológica ${ }^{3}$.

Os membros inferiores (MMII) são responsáveis pela sustentação do peso corporal e da locomoção, estando sujeitos a forças mecânicas internas e externas sobre suas articulações sinoviais, tornando o segmento mais vulnerável a lesão do aparelho locomotor, desencadeando estresses e compensações sobre o seu alinhamento postural. O complexo do joelho, localizado na região intermediária de sobrecarga, é o mais acometido, principalmente durante o apoio unipodal, no qual o desequilíbrio das forças pode desencadear um desalinhamento patológico como alterações ósseas do fêmur, patela e tíbia, além de frouxidão ligamentar excessiva, subluxações e deslocamentos mediais ou laterais (varo e valgo), ou até combinações entre os mesmos ${ }^{4,5}$.
Existem evidências de que o desalinhamento do joelho predispõe a comprometimentos ascendentes e descendentes nas articulações, assim como desequilíbrios musculares e de sobrecarga anormais sobre a articulação do joelho podendo levar a degeneração articular'.

A estabilidade do joelho baseia-se principalmente pelas ações estabilizadoras dos tecidos moles que o cercam, visto que, dois terços dos músculos que cruzam o joeIho também cruzam o quadril e o tornozelo gerando uma forte associação funcional entre essas articulações dos membros inferiores. $\mathrm{O}$ joelho é normalmente alinhado em cerca de 5 a 10 graus de valgismo, essa posição proporciona maior área de superfície articular com o mínimo de estresse possível entre a patela e o fêmur. A compreensão deste alinhamento favorece a interpretação de boa parte da patomecânica e síndromes dolorosas sobre este segmento? ${ }^{7}$.

O chamado valgo dinâmico (VD), termo empregado para descrever a posição do joelho no plano frontal, no qual é determinado pela adução e rotação medial do quadril e abdução e rotação lateral do joelho, ocorre devido esta articulação ser classificada como sinovial gínglimo modificado, visto que na posição de destravamento do joelho o mesmo permite movimentos de rotação, o que impede um adequado alinhamento dinâmico durante a execução de atividades funcionais e um provável motivo relacionado ao surgimento de sintomas álgicos ${ }^{8,9}$.

O valgismo é uma condição favorável para predisposição de lesões no joelho, onde alguns estudos apontam que o deslocamento lateral excessivo é influenciado pela inca- 
pacidade das musculaturas adjacentes em controlar o movimento ${ }^{10,11}$.

A simetria de forças musculares entre agonistas e antagonistas do joelho tornam-se um fator relevante para a manutenção de um alinhamento adequado durante a execução dos movimentos. Músculos uniarticulares, que normalmente possuem função antigravitacional e com predominância de fibras lentas, aparentemente são os mais afetados pelo desuso, causando perda da estabilização do membro inferior, deixando uma grande parte da responsabilidade para os músculos biarticulares, visto que sua biomecânica é mais complexa e possuem dois tempos de contração ${ }^{12}$.

As dores articulares no joelho são vistas como sintomas mais comuns no âmbito da ortopedia e da fisioterapia, afetando principalmente a população feminina sedentária e as praticantes de atividades físicas de impacto, somando os fatores geradores de dores articulares, como afirmam Reis et al. ${ }^{13}$, que esta perturbação neste segmento fica visível em práticas de salto unipodal e bipodal, subir e descer degraus e outras situações que requerem atividades com excesso de descarga de peso no segmento e, indiretamente, oriundos de postura crônica do quadril.

Os sinais e sintomas característicos de patologias que acometem o complexo do joelho, proporcionam interpretações sobre as forças mecânicas atuantes sobre o membro e o comportamento dessas sobre os elementos das regiões adjacentes, construindo relação entre a dinâmica do alinhamento dos MMII e as intervenções direcionadas para minimizar as crises álgicas.

Esse estudo tem relevância em conhe- cer a biomecânica realizada pelos segmentos corpóreos, pois no processo de reabilitação os fundamentos impostos pela mesma favorecem a concepção do processo de diagnóstico diferenciado, que envolve a interpretação das forças que atuam sobre o membro, fazendo com que o registro clínico seja orientado ao problema, o que torna a avaliação fisioterapêutica essencial para compreender a lesão, permitindo a construção de um protocolo de tratamento diferenciado ao paciente, buscando a recuperação da mecânica funcional pré-existente. Desta forma, o objetivo desse estudo foi analisar a biomecânica de membros inferiores em mulheres acometidas com dores articulares no joelho.

\section{METODOLOGIA}

O estudo foi aprovado pelo Comitê de Ética em Pesquisa seguindo as normas da Resolução n . 466/12 do Conselho Nacional de Saúde, relativa à pesquisa em seres humanos, sob o protocolo $n^{\circ}$. 2.013.598 e a assinatura do Termo de Consentimento Livre e Esclarecido (TCLE).

A pesquisa teve uma abordagem observacional analítica e descritiva, do tipo transversal. Participaram do estudo 40 mulheres diagnosticadas com dor articular no joelho e encaminhadas para reabilitação no Centro Clínico Paricuiã, Belém - PA. As participantes foram selecionadas de forma aleatória e avaliadas no período de abril a maio de 2017.

A população em estudo constituiu-se por demanda espontânea, de mulheres com idade entre 18 a 60 anos. Foram incluídas na pesquisa as que possuíam dor articular no joelho e que estivessem, no máximo, 
uma semana de tratamento fisioterapêutico e foram excluídas aquelas que tivessem outras lesões articulares, realizado cirurgia no membro inferior, alterações posturais com diagnóstico clínico, grávidas, aquelas com déficits cognitivos e com patologias neurológicas associadas.

A amostra populacional definiu-se através do número total de pacientes do sexo feminino atendidas nos dois turnos de funcionamento do Centro Clínico, onde em média, são atendidas diariamente 110 mulheres com patologias Traumatológicas e Ortopédicas, variando a quantidade de acordo com a procura do serviço. Destas, 50 tinham problemas relacionados aos joelhos. Houve 10 mulheres que não se enquadraram nos critérios de inclusão e foram excluídas da pesquisa, totalizando 40 participantes no estudo.

Para a coleta dos dados utilizou-se uma ficha de avaliação elaborada pelos pesquisadores, obtendo informações pessoais e clínicas da paciente, estando em ambiente reservado para estes procedimentos iniciais. Após a coleta destas informações, iniciou-se a avaliação estática e dinâmica do segmento acometido. Durante os testes, os participantes pesquisados estavam trajando vestimenta adequada como roupa leve e short, visando melhor observação da cintura pélvica e dos MMII.

Na análise estática do joelho realizou-se uma avaliação biofotogramétrica, por meio do aplicativo SAPO versão 0,69 - maio/2017, que consistiu em elucidar a avaliação postural, por meio de fotos obtidas no plano frontal, pois este analisa fotos digitalizadas dos MMII das voluntárias buscando verificar o comprimento destes, alinhamento pélvico e ângulo Q. Para tal registro, utilizou-se uma câmera fotográfica da marca Sony, modelo DSC-H400 posicionada sobre um tripé de modelo universal, ajustado de acordo com a paciente; a altura foi definida de forma nivelada perpendicularmente à linha horizontal do angulo superior da patela, além da distância da câmera para a paciente, que era de 1,50 metros.

Analisou-se uma foto por vez das investigadas no Software SAPO por meio de projetos independentes, onde foi feito a calibragem vertical e escala (2D) por meio de dois pontos de referência, demarcados na parede ao lado das mulheres pesquisadas, previamente identificados e registrado a distância entre eles. Foi medido o comprimento dos MMII com a opção de medir distância, bem como o alinhamento pélvico e o ângulo $Q$.

Para a análise dinâmica do joelho, utilizou-se o teste Step Down que verificou a presença de valgismo durante uma descida de degrau, onde se capturou o movimento em câmera lenta com o aplicativo de celular chamado SloPro versão 3.6 - março/2016, disponibilizado por Sand Mountain Studios, para registrar a presença ou ausência dessa alteração. Foi utilizado um aparelho celular da marca Apple modelo Iphone 5 para registrar as imagens e uma escada em $L$ de modelo universal, contendo 4 degraus com altura de $10 \mathrm{~cm}$ cada, e $28 \mathrm{~cm}$ entre um degrau e outro.

Foi analisado durante todo o trajeto do teste se ocorreu, em algum momento, alterações no alinhamento do membro, ou seja, do primeiro ao último degrau. No plano frontal, o joelho forma um ângulo no seu aspecto lateral (170 a 175 graus), ângulos menores que esse 
são considerados valgo excessivo, caracterizando a positividade dos testes.

Vale ressaltar o posicionamento dos pés para analise dos resultados, o teste iniciava sempre pelo lado dominante da investigada, mesmo esse apresentando dor, visto que as mesmas se encontravam na fase inicial de tratamento fisioterapêutico. Foram feitas três tentativas para a coleta dos dados e o intervalo entre elas foi apenas o tempo que levavam para voltar à posição inicial e repetir o teste.

O software Microsoft Excel 2010 foi adotado para entrada dos dados, bem como para a confecção das tabelas. A análise estatística foi realizada por meio do software BioEstat 5.0. As variáveis numéricas foram apresentadas por meio de medidas de tendência central e dispersão. Utilizouse o teste D'Agostino para a avaliação da normalidade dos dados. Tratando-se de uma amostra não paramétrica, foram utilizados testes estatísticos apropriados com a característica da amostra. A significância dos dados foi avaliada pelos testes do Quiquadrado (proporções esperadas iguais) e pelo teste de correlação de Spearman. Todos os resultados foram considerados estatisticamente significantes no nível $\alpha$ de $5 \%(p<0,05)$.

\section{RESULTADOS}

A amostra foi composta por 40 mulheres com dor articular no joelho, com média de idade de 45,2 \pm 14,7 anos, média de peso entre as investigadas de $70,3 \pm 12,3 \mathrm{~kg}$, altura de 1,60 \pm 0,05 cm, Índice de Massa Corpórea (IMC) de 27,27 \pm 4,6 e tamanho do pé, segundo o sistema de pontos brasileiro, de $36,5 \pm 1,5 \mathrm{~cm}$. E quanto à posição que adotam durante o trabalho ficou dividido entre as que trabalham em pé $(70 \%)$ e as que trabalham sentadas (30\%), conforme mostra a Tabela 1.

Nos diagnósticos clínicos das pacientes encontra-se uma grande diversidade de patologias, e para classificá-las foram separadas de acordo com o seguimento, como pode ser observado no joelho direto, onde foram encontrados sem lesão (30\%), com Artrite/ Artrose (20\%), Condropatia Patelar (17,5\%),

Tabela 1 - Caracterização (número absoluto e percentual) da população do estudo.

\begin{tabular}{|c|c|c|}
\hline Variáveis & $n=40$ & $\%$ \\
\hline Idade & \multicolumn{2}{|c|}{$45,2 \pm 14,1$} \\
\hline Peso & \multicolumn{2}{|c|}{$70,3 \pm 12,3$} \\
\hline Altura & \multicolumn{2}{|c|}{$1,60 \pm 0,05$} \\
\hline IMC & \multicolumn{2}{|c|}{$27,27 \pm 4,6$} \\
\hline Tamanho do Pé & \multicolumn{2}{|c|}{$36,5 \pm 1,5$} \\
\hline \multicolumn{3}{|c|}{ Posição que adota no trabalho } \\
\hline Trabalha em pé & 28 & $70 \%$ \\
\hline Trabalha sentado & 12 & $30 \%$ \\
\hline
\end{tabular}


Lesão Tendinosa (15\%), Lesão Meniscal (5\%) e Outros (12,5\%). E no joelho esquerdo foi encontrado sem lesão (47,5\%), com Artrite/ Artrose (12,5\%), Condropatia Patelar (12,5\%), Lesão Tendinosa (7,5\%), Lesão Ligamentar (7,5\%), Lesão Meniscal (5\%) e Outros (7,5\%). Vale ressaltar que todas as investigadas obrigatoriamente apresentam lesão em pelo menos um dos joelhos, ou seja, onde se ler na tabela, sem lesão, trata-se do membro sadio e que a lesão se encontrava no membro oposto, conforme mostra a Tabela 2.

Os resultados da avaliação dinâmica feita com o teste Step Down mostraram sig- nificância estatística para os membros inferiores direito das pacientes com o resultado positivo na maioria das investigadas $(77,5 \%$; $p=0,0009$ ), e não mostrou significância nos membros inferiores esquerdo, mesmo obtendo a maioria com o resultado positivo $(65,0 \%$; $p=0,08)$, conforme mostra a Tabela 3 .

Quando se buscou associação das variáveis estudadas com a positividade do teste de Step Down, foi possível inferir que as mesmas mostraram significância estatística com o peso, tanto para o MID $(p=0,05)$ quanto para o esquerdo $(p=0,007)$, assim como para o tamanho do pé do MID $(p=0,04)$ e o IMC

Tabela 2 - Diagnóstico clínico da população do estudo.

\begin{tabular}{l|c|c|c|c}
\hline \multirow{2}{*}{ Diagnóstico clínico } & \multicolumn{2}{c|}{ Joelho Direito } & \multicolumn{2}{c}{ Joelho Esquerdo } \\
\cline { 2 - 5 } & $\mathrm{n}=40$ & $\%$ & $\mathrm{n}=40$ & $\%$ \\
\hline Sem lesão & 12 & $30 \%$ & 19 & $47,5 \%$ \\
Artrite/Artrose & 8 & $20 \%$ & 5 & $12,5 \%$ \\
Condropatia Patelar & 7 & $17,5 \%$ & 5 & $12,5 \%$ \\
Lesão Tendinosa & 6 & $15 \%$ & 3 & $7,5 \%$ \\
Lesão Ligamentar & - & - & 3 & $7,5 \%$ \\
Lesão Meniscal & 2 & $5 \%$ & 2 & $5 \%$ \\
Outros & 5 & $12,5 \%$ & 3 & $7,5 \%$ \\
\hline
\end{tabular}

(-) Dados numéricos igual a zero.

Tabela 3 - Resultado do teste Step Down (número absoluto, percentual e de p-valor) da população do estudo.

\begin{tabular}{lcccc}
\hline Variáveis & $\mathrm{n}=40$ & $\%$ & -valor \\
\hline Step Down D & & & \\
$\quad$ Positivo & 31 & $77,5 \%$ & $0,0009^{*}$ \\
$\quad$ Negativo & 9 & $22,5 \%$ & \\
Step Down E & & & \\
$\quad$ Positivo & 26 & $65,0 \%$ & 0,08 \\
$\quad$ Negativo & 14 & $35,0 \%$ & \\
\hline
\end{tabular}

* Resultado estatisticamente significante. Teste do Qui-quadrado proporções esperadas iguais; $p \leq 0,05$. 
com o MIE $(p=0,01)$. As outras variáveis não se evidenciaram associação estatisticamente significante, conforme mostra a Tabela 4.

Quando pensado a respeito da caracterização postural da amostra obtida por meio da análise estática, chegou-se aos valores de comprimento do MID de $83,5 \pm 5,86 \mathrm{~cm}$, MIE 83,4 \pm 5,89cm, ângulo Q do MID 25,2 \pm 6,09 graus, ângulo $Q$ do MIE 25,3 $\pm 6,57$ graus e inclinação pélvica de 1,20 $\pm 0,89 \mathrm{~cm}$. Ao avaliar para qual lado a inclinação pélvica era mais prevalente, a partir do número absoluto e percentagem, foi identificado que houve 9 inclinações para a direita $(22,5 \%), 25$ para a esquerda $(62,5 \%)$ e 6 alinhados e classificados como neutro (15\%), de acordo como mostra a Tabela 5.

$\mathrm{Na}$ correlação dos dados posturais com a avaliação dinâmica, o teste de Step Down mostrou significância estatística em uma das variáveis do estudo, o comprimento do MID $(p=0,01)$. As demais variáveis não apresentaram valores significantes estatisticamente, como pode ser visto na Tabela 6 .

\section{DISCUSSÃO}

Os valores que indicam alta correlação do valgo dinâmico com o resultado positivo do Teste Step Down (TSD) nas mulheres que participaram do estudo, podem ser originados pela fraqueza de grupos musculares e de sua capacidade de gerar torque nos membros inferiores. Em apoio a isto, Burnham et al. ${ }^{14}$ em seu estudo relataram que na análise univariada dos seus participantes obtiveram correlação positiva estatisticamente significante com o desempenho do TSD relacionada positivamente com a função muscular do quadril e do tronco e, além disso, que essas afinidades foram mais fortes em mulheres.

Tabela 4 - Correlação dos dados demográficos e o teste Step Down da população do estudo, $n=40$

\begin{tabular}{lcc}
\hline Variáveis & $\mathrm{R}$ & $\mathrm{p}$-valor \\
\hline Idade X Step Down D & 0,20 & 0,20 \\
Idade X Step Down E & 0,25 & 0,11 \\
Peso X Step Down D & 0,30 & $0,05^{*}$ \\
Peso X Step Down E & 0,41 & $0,007^{*}$ \\
Altura X Step Down D & 0,22 & 0,15 \\
Altura X Step Down E & 0,00 & 0,96 \\
Tamanho do Pé X Step Down D & 0,31 & $0,04^{*}$ \\
Tamanho do Pé X Step Down E & 0,02 & 0,89 \\
IMC X Step Down D & 0,24 & 0,12 \\
IMC X Step Down E & 0,38 & $0,01^{*}$ \\
Profissão X Step Down D & 0,22 & 0,16 \\
Profissão X Step Down E & 0,13 & 0,39 \\
\hline
\end{tabular}

* Resultado estatisticamente significante. Teste de correlação de Spearman, $p \leq 0,05$.

$\mathrm{R}=$ Coeficiente de Spearman 
Tabela 5 - Caracterização postural da população do estudo.

\begin{tabular}{|c|c|c|}
\hline Variáveis & $\mathrm{n}=40$ & $\%$ \\
\hline \multicolumn{3}{|l|}{ Comprimento } \\
\hline Membro Inferior Direito (MID) & \multicolumn{2}{|c|}{$83,5 \pm 5,86$} \\
\hline Membro Inferir Esquerdo (MIE) & \multicolumn{2}{|c|}{$83,4 \pm 5,89$} \\
\hline \multicolumn{3}{|l|}{ Ângulo Q } \\
\hline Membro Inferior Direito (MID) & \multicolumn{2}{|c|}{$25,2 \pm 6,09$} \\
\hline Membro Inferir Esquerdo (MIE) & \multicolumn{2}{|c|}{$25,3 \pm 6,57$} \\
\hline Inclinação Pélvica & \multicolumn{2}{|c|}{$1,20 \pm 0,89$} \\
\hline \multicolumn{3}{|l|}{ Lado da Inclinação Pélvica } \\
\hline Direito & 9 & $22,5 \%$ \\
\hline Esquerdo & 25 & $62,5 \%$ \\
\hline Neutro & 6 & $15 \%$ \\
\hline
\end{tabular}

Tabela 6 - Correlação dos dados posturais e o teste Step Down da população do estudo, $\mathrm{n}=$ 40.

\begin{tabular}{lcc}
\hline Variáveis & $\mathrm{R}$ & $\mathrm{p}$-valor \\
\hline Comprimento MID X Step Down D & 0,39 & $0,01^{*}$ \\
Comprimento MIE X Step Down E & $-0,01$ & 0,91 \\
Ângulo Q MID X Step Down D & 0,07 & 0,66 \\
Ângulo Q MIE X Step Down E & 0,11 & 0,47 \\
Inclinação Pélvica X Step Down D & 0,11 & 0,46 \\
Inclinação Pélvica X Step Down E & $-0,26$ & 0,09 \\
\hline
\end{tabular}

* Resultado estatisticamente significante. Teste de correlação de Spearman, $p \leq 0,05$.

MID = Membro Inferior Direito

MIE = Membro Inferior Esquerdo

$\mathrm{R}=$ Coeficiente de Spearman

A grande relação do peso corporal com o valgismo de joelho foi identificada nos resultados com significância para ambos os membros, fomentando a hipótese de que, possivelmente, as mulheres com peso, em média, de $70,3 \mathrm{~kg}$ desenvolvem a medialização da articulação do joelho devido à força de reação que o solo exerce contrária ao membro inferior, associado também a outras variáveis. Diante disso, Fabris et al..$^{15}$ confir- maram esse raciocínio quando em seu estudo buscou analisar por meio da baropodometria em indivíduos obesos e descobriu que o pico de pressão plantar, a força de reação do solo e a região de contato do pé com o solo são maiores do que em pessoas que não apresentam sobrepeso ou obesidade.

Acredita-se que o peso corporal isolado, assim como o IMC em valores altos, que está associado à obesidade, é um dos 
preditores para o mau alinhamento do membro inferior, onde durante a realização de atividade ativa existe grande possibilidade desse desalinhamento ser mais aparente. Nos dados deste estudo, foi encontrada a relação do IMC com o valgismo dinâmico no MIE, sendo confirmada nos resultados do trabalho de Bout-Tabaku et al. ${ }^{16}$ onde usaram 155 pessoas saudáveis e 165 obesos e avaliaram o alinhamento do joelho desses indivíduos confrontando os resultados dos grupos, tendo encontrado maior variabilidade significativamente no alinhamento do joelho entre as mulheres com maiores escores de IMC, levando-se a supor que quanto maior for o valor do IMC, maior será as chances de surgir valgo de joelho em mulheres.

O ângulo $Q$ da análise postural não teve significância quando correlacionada com a presença do valgo dinâmico, isso mostra que nas participantes do estudo a magnitude do ângulo $Q$ não interferiria no alinhamento dinâmico dos joelhos ${ }^{17}$. Corroborando com esses resultados, o estudo de Almeida et al. ${ }^{18}$ onde foram avaliadas 22 mulheres, tendo sido identificado que pacientes com aumento do ângulo $Q$ não tiveram valgismo acentuado no joelho.

O pé é a base de sustentação do corpo e um elemento importante a ser questionado quando se trata de lesões no joelho, principalmente porque os resultados do estudo encontraram relação entre o tamanho dos pés com a presença do valgo dinâmico. Sabe-se que a relação do tamanho da base de sustentação está associada ao melhor controle para estabilização do seguimento e mudanças de movimentos e má posturas adotadas influenciam na mecânica articular do joelho. Com base nessa afirmação, Golias ${ }^{19}$ ao pesquisar essa relação em 21 atletas de ginástica rítmica relata que a movimentação da cadeia cinética da extremidade inferior na marcha se correlaciona com as disfunções do pé com distúrbios no joelho, concluindo que a posição e o movimento do pé como fator intrínseco, podem causar lesões no joelho por uso excessivo.

Não foram achados na literatura outros trabalhos que identificassem a relação do comprimento do membro inferior com a presença de valgo de joelho, dificultando manter a hipótese de que um membro inferior, em média, com $83,5 \mathrm{~cm}$ de comprimento, pode ser um facilitador do acometimento do valgo dinâmico em mulheres que sentem dor articular no joelho.

Quando investigado a presença de inclinação pélvica associada ao valgismo não foi identificada significância estatística, mesmo que obtidos valores afastados do normal. Controverso a esse achado, Guimarães et al. ${ }^{20}$ relataram em seu estudo, realizado com 38 praticantes de ginástica olímpica, que o aumento da inclinação pélvica e a disposição ao aumento da hiperlordose lombar podem predispor às lesões futuras.

Ainda é escassa a produção científica relacionando a biomecânica com a prática clínica, com o intuito de melhorar o manejo terapêutico do paciente. Desta maneira, este estudo mostrou que metodologias relativamente simples e acessíveis podem contribuir para o aprimoramento de uma fisioterapia baseada em evidências.

Embora tenha conseguido, neste estudo, resultados potencialmente positivos, ressalta-se que é de grande importância que sejam desenvolvidas mais pesquisas que avaliem cuidadosamente os processos bio- 
mecânicos relacionados ao desenvolvimento de lesões articulares no joelho. Salienta-se ainda, que existem limitações no que se refere à metodologia aplicada, visto que os testes utilizados ainda possuem uma análise subjetiva do avaliador, pontos estes, que podem servir de base para novas pesquisas.

\section{CONCLUSÃO}

$\mathrm{Na}$ análise biomecânica foi possível encontrar que as variáveis peso corporal, IMC, tamanho do pé e o comprimento do membro inferior tiveram relação direta sobre o quadril e os MMII, sugerindo o valgo dinâmico e/ou predisposição do agravamento de dores articulares no joelho em mulheres.

O teste Step Down mostrou-se excelente medidor do valgo dinâmico de joelho, pois a execução é rápida e dinâmica, além de ser possível tê-lo como base para a prescrição dos exercícios terapêuticos mais apropriados, otimizando o tratamento e os resultados futuros.

\section{REFERÊNCIAS}

1. Nobre TL. Comparação dos exercícios em cadeia cinética aberta e cadeia cinética fechada na reabilitação da disfunção femoropatelar. Fisioter. Mov. 2011;24(1):167172.

2. Liporaci RF, Saad MC, Felício LR, Baffa AP, Grossi DB. Contribuição da avaliação dos sinais clínicos em Pacientes com síndrome da dor patelofemoral. Acta Ortop Bras. 2013;21(4):198-201.

3. Dugan SA. Sports-Related Knee Injuries in Female Athletes. What Gives? Am. J. Phys. Med. Rehabil. 2005;84(2):122-130.

4. Hebert S, Barros Filho TEP, Xavier R, Pardini JR. Ortopedia e Traumatologia: princípios e prática. $4^{a}$.ed. Porto Alegre: Editora Artmed; 2009.

5. Ginnis PM. Biomecânica do esporte e do exercício. $3^{\circ}$.ed. Rio de Janeiro: Artmed, 2015.

6. Oatis CA. Cinesiologia: a mecânica e a patomecânica do movimento humano. $2^{\circ}$.ed. São Paulo: Manole, 2014.

7. Neumann DA. Cinesiologia do aparelho musculoesquelético. $2^{\circ}$.ed. Rio de Janeiro: Elsevier, 2011.

8. Queiroz A, Atalaia T, Coutinho Ml. Ativação muscular na anca e joelho na variação do ângulo de valgo durante a fase de apoio do salto vertical. Saúde \& Tecnologia. 2014; 12:8-16

9. Oliveira DS, Briani MRP, Ferrari FDA, Azevedo F. Vertical Ground Reaction Forces are Associated with Pain and Self-Reported Functional Status in Recreational Athletes with Patellofemoral Pain. J Appl Biomech. 2015;31(6):409414.

10. Baldon RM, Lobato DFM, Carvalho LP, Wun PYL, Serrão FV. Diferenças biomecânicas entre os gêneros e sua importância nas lesões do joelho. Fisioter. Mov. 2011;24(1):157-166.

11. Hall SJ. Biomecânica Básica. 6a ${ }^{a}$.ed. Rio de Janeiro: Guanabara Koogan; 2013.

12. Baroni $B M$, Galvão $A Q$, Ritzel $C H$, Diefenthaeler $F$, Vaz MA. Adaptações Neuromusculares de Flexores Dorsais e Plantares a Duas Semanas de Imobilização Após Entorse de Tornozelo. Rev Bras Med Esporte. 2010;16(5):358-362.

13. Reis AC, Bley AS, Rabelo NDA, Castilho A, Fukuda TY, Lucareli PRG. Características cinemáticas do salto de mulheres com síndrome da dor femoropatelar. Fisioterapia Brasil. 2013;15(3):224-227.

14. Burnham JM, Yonz MC, Robertson KE, Mckinley R, Wilson BR, Johnson DL, et al. Relationship of Hip and Trunk Muscle Function with Single Leg Step-Down Performance. Physical Therapy in Sport. 2016; 22:66-73.

15. Fabris MS, Valezi AC, Souza SAF, Faintuch J, Cecconello I, Junior MP. Computerized Baropodometry In Obese Patients. Obesity Surgery. 2006;16(12):1574-1578.

16. Bout-Tabaku S, Shults J, Zemel BS, Leonard MB, Berkowitz RI, Stettler N, Burnham JM. Obesity Is Associated with Greater Valgus Knee Alignment in Pubertal Children, and Higher Body Mass Index Is Associated with Greater Variability in Knee Alignment in Girls. The Journal of Rheumatology. 2014;42(1):1-8.

17. Bini RR, Carpes FP, Toledo JM, Loss JF. Estimativa das forças na articulação tíbio-femoral no exercício de extensão dos joelhos em cadeia cinética aberta realizado em máquina de musculação. Rev. Bras. Cine Antropom. Desempenho Hum. 2008;10(1):35-42.

18. Almeida GPL, Silva APMCC, Franca FJR, Magalhães $\mathrm{MO}$, Burke TN, Marques AP. Ângulo-Q na dor patelofemoral: relação com valgo dinâmico de joelho, torque abdutor do quadril, dor e função. Rev. Brasileira de Ortopedia. 2016;51(2):181-186.

19. Golias ARC. Relação entre instabilidade do joelho e alterações posicionais do membro inferior de atletas de Ginástica Rítmica. R. Bras. Cien. e Mov. 2012;20(2):5260.

20. Guimarães MMB, Sacco ICN, João SMA. Postura characterization of young participants in Olympic gymnastics. Rev. Bras. Fisioter. 2007;11(3):213-219.

\section{CORRESPONDÊNCIA}

George Alberto da Silva Dias

Universidade do Estado do Pará (UEPA), Rua do Una, 156 Belém - Pará - Brasil - CEP: 66050540 - Telegrafo george@uepa.br 